Korean J. Math. 19 (2011), No. 3, pp. 331-342

\title{
AT LEAST TWO SOLUTIONS FOR THE ASYMMETRIC BEAM SYSTEM WITH CRITICAL GROWTH
}

\author{
Tacksun Jung And Q-Heung Choi*
}

Abstract. We consider the multiplicity of the solutions for a class of a system of critical growth beam equations with periodic condition on $t$ and Dirichlet boundary condition

$$
\left\{\begin{aligned}
u_{t t}+u_{x x x x}=a v+\frac{2 \alpha}{\alpha+\beta} u_{+}^{\alpha-1} v_{+}^{\beta}+s \phi_{00} & \text { in }\left(-\frac{\pi}{2}, \frac{\pi}{2}\right) \times R, \\
v_{t t}+v_{x x x x}=b u+\frac{2 \beta}{\alpha+\beta} u_{+}^{\alpha} v_{+}^{\beta-1}+t \phi_{00} & \text { in }\left(-\frac{\pi}{2}, \frac{\pi}{2}\right) \times R,
\end{aligned}\right.
$$

where $\alpha, \beta>1$ are real constants, $u_{+}=\max \{u, 0\}, \phi_{00}$ is the eigenfunction corresponding to the positive eigenvalue $\lambda_{00}=1$ of the eigenvalue problem $u_{t t}+u_{x x x x}=\lambda_{m n} u$. We show that the system has a positive solution under suitable conditions on the matrix $A=\left(\begin{array}{ll}0 & a \\ b & 0\end{array}\right), s>0, t>0$, and next show that the system has another solution for the same conditions on $A$ by the linking arguments.

\section{Introduction}

McKenna and Walter [4] found the physical model of jumping problem from a bridge suspended by cables under a load. The nonlinear suspension bridge equation (with length $\pi$ ) is as follows

$$
u_{t t}+u_{x x x x}+b u^{+}=f(x, t) \quad \text { in }\left(-\frac{\pi}{2}, \frac{\pi}{2}\right) \times R,
$$

Received August 5, 2011. Revised September 15, 2011. Accepted September 20, 2011.

2000 Mathematics Subject Classification: 35Q72.

Key words and phrases: system of suspension bridge equations, critical growth, linking arguments, eigenvalue of a matrix, boundary value problem.

This work(Choi) was supported by Basic Science Research Program through the National Research Foundation of Korea(NRF) funded by the Ministry of Education, Science and Technology (KRF-2009-0071291).

${ }^{*}$ Corresponding author. 


$$
u\left( \pm \frac{\pi}{2}, t\right)=u_{x x}\left( \pm \frac{\pi}{2}, t\right)=0
$$

$$
u \text { is } \pi \text {-periodic in } t \text { and even in } x \text { and } t \text {, }
$$

where the nonlinearity $-\left(b u^{+}\right)$crosses an eigenvalue $\lambda_{10}$. This equation represents a bending beam supported by cables under a load $f$. The constant $b$ represents the restoring force if the cables stretch. The nonlinearity $u^{+}$models the fact that cables resist expansion but do not resist compression.

Let $L$ be the differential operator, $L u=u_{t t}+u_{x x x x}$. Then the eigenvalue problem for $u(x, t)$

$$
L u=\lambda u \quad \text { in }\left(-\frac{\pi}{2}, \frac{\pi}{2}\right) \times R
$$

with (1.2) and (1.3) has infinitely many eigenvalues

$$
\lambda_{m n}=(2 n+1)^{4}-4 m^{2} \quad(m, n=0,1,2, \cdots)
$$

and corresponding eigenfunctions $\phi_{m n}(m, n \geq 0)$ given by

$$
\phi_{m n}=\cos 2 m t \cos (2 n+1) x .
$$

We note that all eigenvalues in the interval $(-19,45)$ are given by

$$
\lambda_{20}=-15<\lambda_{10}=-3<\lambda_{00}=1<\lambda_{41}=17 .
$$
by

Let $Q$ be the square $\left[-\frac{\pi}{2}, \frac{\pi}{2}\right] \times\left[-\frac{\pi}{2}, \frac{\pi}{2}\right]$ and $H$ the Hilbert space defined

$$
H=\left\{u \in L^{2}(Q) \mid u \text { is even in } x \text { and } t\right\} .
$$

Then the set of eigenfunctions $\left\{\phi_{m n}\right\}$ is an orthonormal base in $H$. Hence equation (1.1) with (1.2) and (1.3) is equivalent to

$$
L u+b u^{+}=f \quad \text { in } H .
$$

McKenna and Walter [4] showed by degree theory that equation (1.4) with constant load $1+\epsilon h$ ( $h$ is bounded ) has at least two solutions.

Choi, Jung and McKenna [1] showed by a variational reduction method that equation (1.4) with constant load $1+\epsilon h$ ( $h$ is bounded ) has at least three solutions when condition (1.3) is replaced by

$$
u \text { is } \pi \text {-periodic in } t \text { and even in } x \text {. }
$$

Liu [3] consider the semilinear beam equation where the nonlinear term is a function with different powers. 
At least two solutions for the asymmetric beam system with critical growth 333

A typical example is

$$
f(x, t, s)= \begin{cases}s^{2}, & s \geq 0 \\ s^{3}, & s \leq 0 .\end{cases}
$$

Nam and Choi [5] considered the following beam equation (1)

$$
\begin{gathered}
u_{t t}+u_{x x x x}+b u^{+}=f(x, t, u) \quad \text { in }\left(-\frac{\pi}{2}, \frac{\pi}{2}\right) \times R, \\
u\left( \pm \frac{\pi}{2}, t\right)=u_{x x}\left( \pm \frac{\pi}{2}, t\right)=0,
\end{gathered}
$$

$\mathrm{u}$ is $\pi$-periodic in $\mathrm{t}$ and even in $\mathrm{x}$ and $\mathrm{t}$,

where $f$ is defined by

(2)

$$
f(x, t, s)= \begin{cases}|s|^{p-2} s, & s \geq 0 \\ |s|^{q-2} s, & s<0\end{cases}
$$

where $p, q>2$ and $p \neq q$.

We denote by $\left(\Lambda_{i}^{-}\right)_{i \geq 1}$ the sequence of the negative eigenvalues of eigenvalue problem $L u=\lambda u$, by $\left(\Lambda_{i}^{+}\right)_{i \geq 1}$ the sequence of the positive ones, so that

$$
\cdots<\Lambda_{1}^{-}=-3<\Lambda_{1}^{+}=1<\Lambda_{2}^{+}=17<\cdots .
$$

We consider an orthonormal system of eigenfunctions $\left\{e_{i}^{-}, e_{i}^{+}, i \geq 1\right\}$ associated with the eigenvalues $\left\{\Lambda_{i}^{-}, \Lambda_{i}^{+}, i \geq 1\right\}$.

Nam and Choi [5] show by linking theorem that if $\Lambda_{i}^{-} \leq-b$ then problem (1) has at least one nontrivial solution. They [5] also show by category theory that problem (1) has at least three nontrivial solutions if $i \geq 2$.

In this paper we consider the multiplicity of the solutions for a class of a system of critical growth beam equations, with periodic condition on $t$ and Dirichlet boundary condition

$$
\text { (1.5) } \begin{cases}u_{t t}+u_{x x x x} & =a v+\frac{2 \alpha}{\alpha+\beta} u_{+}^{\alpha-1} v_{+}^{\beta}+s \phi_{00} \quad \text { in }\left(-\frac{\pi}{2}, \frac{\pi}{2}\right) \times R \\ v_{t t}+v_{x x x x} & =b u+\frac{2 \beta}{\alpha+\beta} u_{+}^{\alpha} v_{+}^{\beta-1}+t \phi_{00} \quad \text { in }\left(-\frac{\pi}{2}, \frac{\pi}{2}\right) \times R \\ u\left( \pm \frac{\pi}{2}, t\right) & =u_{x x}\left( \pm \frac{\pi}{2}, t\right)=v\left( \pm \frac{\pi}{2}, t\right)=v_{x x}\left( \pm \frac{\pi}{2}, t\right)=0 \\ u(x, t+\pi) & =u(x, t)=u(-x, t)=u(x,-t) \\ v(x, t+\pi) & =v(x, t)=v(-x, t)=v(x,-t)\end{cases}
$$


where $\alpha, \beta>1$ are real constants, $u_{+}=\max \{u, 0\}, \phi_{00}$ is the eigenfunction corresponding to the positive eigenvalue $\lambda_{00}=1$ of the eigenvalue problem $u_{t t}+u_{x x x x}=\lambda_{m n} u$ with (1.2), (1.3).

The system can be rewritten by

$$
\begin{cases}\mathcal{L} U & =\nabla\left(\frac{1}{2}(A U, U)+\frac{2}{\alpha+\beta} u_{+}^{\alpha} v_{+}^{\beta}\right)+\left(\begin{array}{c}
s \phi_{00} \\
t \phi_{00}
\end{array}\right) \\
U\left( \pm \frac{\pi}{2}, t\right) & =U_{x x}\left( \pm \frac{\pi}{2}, t\right)=\left(\begin{array}{l}
0 \\
0
\end{array}\right) \\
U(x, t+\pi) & =U(x, t)=U(-x, t)=U(x,-t)\end{cases}
$$

where $\nabla$ is the gradient operator, $U=\left(\begin{array}{l}u \\ v\end{array}\right), \mathcal{L} U=\left(\begin{array}{l}L u \\ L v\end{array}\right), L u=u_{t t}-u_{x x}$, $A=\left(\begin{array}{cc}0 & a \\ b & 0\end{array}\right) \in M_{2 \times 2}(R)$.

We assume that

$$
\begin{aligned}
& \lambda_{m n}^{2}-a b \neq 0 \text { for all } m, n \text { with }(m, n) \neq(0,0), \\
& \qquad \begin{array}{c}
a>0, \quad b>0, \\
\sqrt{a b}<1 .
\end{array}
\end{aligned}
$$

Our main result is the following:

Theorem 1.1. Assume that the conditions (A), (B) and (C) hold. Then for $(s, t)$ with $s>0$ and $t>0$ system (1.5) has at least two solutions, one of which is a positive solution $U_{0}=\left(\begin{array}{l}u_{0} \\ v_{0}\end{array}\right)$ with $u_{0}>0$ and $v_{0}>0$.

In section 2, we show that system (1.5) has a negative solution. In section 3 , we approach the variational method and recall the critical point theorem which is the linking theorem for the strongly indefinite functional to find the second solution. In section 4, we prove the existence of the second solution of (1.5).

\section{Linear problem}

The eigenvalue problem $L u=\lambda u$ with (1.2), (1.3) has infinitely many eigenvalues

$$
\lambda_{m n}=(2 n+1)^{4}-4 m^{2} \quad(m, n=0,1,2, \ldots)
$$

and corresponding normalized eigenfunctions $\phi_{m n}(m, n \geq 0)$ given by

$$
\phi_{0 n}=\frac{\sqrt{2}}{\pi} \cos (2 n+1) x \quad \text { for } n \geq 0 \text {, }
$$


At least two solutions for the asymmetric beam system with critical growth 335

$$
\phi_{m n}=\frac{2}{\pi} \cos 2 m t \cdot \cos (2 n+1) x \quad \text { for } m>0, n \geq 0 .
$$

Let $Q$ be the square $\left[-\frac{\pi}{2}, \frac{\pi}{2}\right] \times\left[-\frac{\pi}{2}, \frac{\pi}{2}\right]$ and $H_{0}$ the Hilbert space defined by

$$
H_{0}=\left\{u \in L^{2}(Q) \mid u \text { is even in } x \text { and } t\right\} .
$$

The set of functions $\left\{\phi_{m n}\right\}$ is an orthonormal basis in $H_{0}$. Let us denote an element $u$ in $H_{0}$ by

$$
u=\sum h_{m n} \phi_{m n}
$$

We define a Hilbert space $\mathcal{D}$ as follows

$$
\mathcal{D}=\left\{u \in \sum h_{m n} \phi_{m n} \mid \sum_{m n} \lambda_{m n}^{2} h_{m n}^{2}<+\infty\right\} .
$$

Then this space is a Banach space with norm

$$
\|u\|=\left[\sum \lambda_{m n}^{2} h_{m n}^{2}\right]^{\frac{1}{2}}
$$

Let us set $E=\mathcal{D} \times \mathcal{D}$. We endow the Hilbert space $E$ with the norm

$$
\|(u, v)\|_{E}^{2}=\|u\|^{2}+\|v\|^{2} .
$$

We are looking for the weak solutions of $(1.5)$ in $\mathcal{D} \times \mathcal{D}$. We have some properties. Since $\left|\lambda_{m n}\right| \geq 1$ for all $m$, $n$, we have the following lemma (cf. [2]).

Lemma 2.1. (i) $\|u\| \geq\|u\|_{L^{2}(Q)}$, where $\|u\|_{L^{2}(Q)}$ denotes the $L^{2}$ norm of $u$.

(ii) $\|u\|=0$ if and only if $\|u\|_{L^{2}(Q)}=0$.

(iii) $u_{t t}+u_{x x x x} \in \mathcal{D}$ implies $u \in \mathcal{D}$.

Lemma 2.2. Suppose that $c$ is not an eigenvalue of $L: \mathcal{D} \rightarrow H_{0}$, $L u=u_{t t}-u_{x x}$, and let $f \in H_{0}$. Then we have $(L-c)^{-1} f \in \mathcal{D}$.

Proof. Let $\lambda_{m n}$ is an eigenvalue of $L$. We notice that $\left\{\lambda_{m n}|| \lambda_{m n} \mid<\right.$ $|c|\}$ is finite. Let

$$
f=\sum h_{m n} \phi_{m n}
$$

Then

$$
(L-c)^{-1} f=\sum \frac{1}{\lambda_{m n}-c} h_{m n} \phi_{m n}
$$


Hence we have the inequality

$$
\left\|(L-c)^{-1} f\right\|^{2}=\sum \lambda_{m n}^{2} \frac{1}{\left(\lambda_{m n}-c\right)^{2}} h_{m n}^{2} \leq C \sum h_{m n}^{2}
$$

for some $C$, which means that

$$
\left\|(L-c)^{-1} f\right\| \leq C_{1}\|f\|_{L^{2}(Q)}, \quad C_{1}=\sqrt{C} .
$$

Lemma 2.3. Assume that the conditions $(A),(B)$ and $(C)$ hold. Then the system

$$
\begin{cases}u_{t t}+u_{x x x x} & =a v+s \phi_{00} \quad \text { in }\left(-\frac{\pi}{2}, \frac{\pi}{2}\right) \times R \\ v_{t t}+v_{x x x x} & =b u+t \phi_{00} \quad \text { in }\left(-\frac{\pi}{2}, \frac{\pi}{2}\right) \times R \\ u\left( \pm \frac{\pi}{2}, t\right) & =u_{x x}\left( \pm \frac{\pi}{2}, t\right)=v\left( \pm \frac{\pi}{2}, t\right)=v_{x x}\left( \pm \frac{\pi}{2}, t\right)=0 \\ u(x, t+\pi) & =u(x, t)=u(-x, t)=u(x,-t) \\ v(x, t+\pi) & =v(x, t)=v(-x, t)=v(x,-t)\end{cases}
$$

has a unique solution $\left(u_{*}, v_{*}\right) \in E=\mathcal{D} \times \mathcal{D}$, which is of the form

$$
u_{*}=\left[\frac{s+a t \lambda_{00}}{\lambda_{00}^{2}-a b}\right] \phi_{00}, v_{*}=\left[\frac{b s+t \lambda_{00}}{\lambda_{00}^{2}-a b}\right] \phi_{00} .
$$

Proof. We note that $\left(u_{*}, v_{*}\right)$ is a solution of system (2.1) and the uniqueness is the consequence of Lemma 2.4 and Lemma 2.5.

We need to find a spectral analysis for the linear operator $\mathcal{L} U-A U$. The following lemma need a simple 'Fourier Series' argument(cf. [2]).

Lemma 2.4. Let $a, b \in R$ and let $\mathcal{L}_{a b}: \mathcal{D} \times \mathcal{D} \rightarrow H_{0} \times H_{0}$ be defined by $\mathcal{L}_{a b}(u, v)=(L u-a v, L v-b u)$. For $\mu \in R$ we have

(a) if $\left(\lambda_{m n}-\mu\right)^{2} \neq a b$ for every $m, n$, then

$$
\left(\mathcal{L}_{a b}-\mu I\right)^{-1} \mid H_{0} \times H_{0} \rightarrow H_{0} \times H_{0}
$$

is well defined and continuous;

(b) if $\left(\lambda_{m n}-\mu\right)^{2}=a b$ for some $m, n$, then

$$
\operatorname{Ker}\left(\mathcal{L}_{a b}-\mu I\right)=\operatorname{span}\left\{\phi_{m n} \mid\left(\lambda_{m n}-\mu\right)^{2}=a b\right\} ;
$$

moreover if $X_{\mu}=\overline{\operatorname{span}\left\{\phi_{m n} \mid\left(\lambda_{m n}-\mu\right)^{2} \neq a b\right\}}$, then

$$
\left(\mathcal{L}_{a b}-\mu I\right)^{-1} \mid X_{\mu} \times X_{\mu} \rightarrow X_{\mu} \times X_{\mu}
$$

is well defined and continuous.

Notice that if $a b<0$, the second alternative can never occur. 
At least two solutions for the asymmetric beam system with critical growth 337

Using Lemma 2.4 with the case $\mu=0$ we can easily derive Lemma 2.5 .

Lemma 2.5. Assume that the conditions (A), (B) and (C) hold. Then the system

$$
\begin{gathered}
\mathcal{L} U-A U=0, \quad U=\left(\begin{array}{l}
u \\
v
\end{array}\right) \in E, \\
U\left( \pm \frac{\pi}{2}, t\right)=U_{x x}\left( \pm \frac{\pi}{2}, t\right)=0, \\
U(x, t+\pi)=U(x, t)=U(-x, t)=U(x,-t)
\end{gathered}
$$

has only the trivial solution $U(x, t)=\left(\begin{array}{l}0 \\ 0\end{array}\right)$.

\section{Variational approach}

In this section we suppose that $s>0, t>0$. Then (1.5) has a positive solution $\left(u_{0}, v_{0}\right)$ with $u_{0}>0, v_{0}>0$. To find the second solution of system (1.5) we approach the variational method and recall the linking theorem for the strongly indefinite functional. Now we are looking for the weak solutions of system (1.5). We observe that the weak solutions of (1.5) coincide with the critical points of the corresponding functional $I: E \rightarrow R \in C^{1,1}$,

$$
\begin{aligned}
I(U)= & \frac{1}{2} \int_{Q} \mathcal{L} U \cdot U d x d t-\frac{1}{2} \int_{Q}(A U, U)_{R^{2}} d x d t \\
& -\frac{2}{\alpha+\beta} \int_{Q} u_{+}^{\alpha} v_{+}^{\beta} d x d t-\int_{Q} s \phi_{00} u d x d t-\int_{Q} t \phi_{00} v d x d t
\end{aligned}
$$

We notice that the solution $(u, v)$ of system $(1.5)$ is of the form $(u, v)=$ $(\bar{u}, \bar{v})+\left(u_{0}, v_{0}\right)$, where $(\bar{u}, \bar{v})$ is a nontrivial solution of the system

$$
\left\{\begin{array}{c}
u_{t t}-u_{x x}=a v+\frac{2 \alpha}{\alpha+\beta}\left(u+u_{0}\right)_{+}^{\alpha-1}\left(v+v_{0}\right)_{+}^{\beta} \\
\text { in }\left(-\frac{\pi}{2}, \frac{\pi}{2}\right) \times\left(-\frac{\pi}{2}, \frac{\pi}{2}\right), \\
v_{t t}-v_{x x}=b u+\frac{2 \beta}{\alpha+\beta}\left(u+u_{0}\right)_{+}^{\alpha}\left(v+v_{0}\right)_{+}^{\beta-1} \\
\text { in }\left(-\frac{\pi}{2}, \frac{\pi}{2}\right) \times\left(-\frac{\pi}{2}, \frac{\pi}{2}\right), \\
u\left( \pm \frac{\pi}{2}, t\right)=u_{x x}\left( \pm \frac{\pi}{2}, t\right)=v\left( \pm \frac{\pi}{2}, t\right)=v_{x x}\left( \pm \frac{\pi}{2}, t\right)=0 \\
u(x, t+\pi)=u(x, t)=u(-x, t)=u(x,-t) \\
v(x, t+\pi)=v(x, t)=v(-x, t)=v(x,-t)
\end{array}\right.
$$


Thus it suffices to find the nontrivial solution of system (3.2). We observe that the weak solutions of (3.2) are the critical points of the functional $J: E \rightarrow R \in C^{1,1}$,

$$
\begin{aligned}
J(U)=\frac{1}{2} \int_{Q} \mathcal{L} U & \cdot U d x d t-\frac{1}{2} \int_{Q}(A U, U)_{R^{2}} d x d t \\
& -\frac{2}{\alpha+\beta} \int_{Q}\left(u+u_{0}\right)_{+}^{\alpha}\left(v+v_{0}\right)_{+}^{\beta} d x d t .
\end{aligned}
$$

Thus we shall find the critical points for $J$. Now we recall the linking theorem for strongly indefinite functional (cf. [6]).

Lemma 3.1. (Linking Theorem)

Let $E$ be a real Hilbert space with $E=E_{1} \oplus E_{2}$ and $E_{2}=E_{1}^{\perp}$. We suppose that

(J1) $J \in C^{1}(E, R)$ satisfies (P.S.) condition,

(J2) $J(u)=\frac{1}{2}(L u, u)+b u$, where $L u=L_{1} P_{1} u+L_{2} P_{2} u$ and $L_{i}: E_{i} \rightarrow E_{i}$ is bounded and selfadjoint, $i=1,2$,

(J3) $b^{\prime}$ is compact,

(J4) there exists a subspace $\tilde{E} \subset E$ and sets $S \subset E, T \subset \tilde{E}$ and constants $\gamma>w$ such that

(i) $S \subset E_{1}$ and $\left.J\right|_{S} \geq \gamma$,

(ii) $T$ is bounded and $\left.J\right|_{\partial T} \leq w$,

(iii) $S$ and $\partial T$ link.

Then $J$ possesses a critical value $c \geq \gamma$.

Let $E^{+}, E^{0}, E^{-}$be the subspace of $E$ on which the functional $U \mapsto$ $\frac{1}{2} \int_{Q} \mathcal{L} U \cdot U$ is positive definite, null, negative definite, respectively and $E^{+}, E^{0}$ and $E^{-}$are mutually orthogonal. Let $P^{+}$be the projection for $E$ onto $E^{+}, P^{0}$ the one from $E$ onto $E^{0}$ and $P^{-}$the one from $E$ onto $E^{-}$. Let $\left(E_{n}\right)_{n}$ be a sequence of closed subspaces of $E$ with the conditions:

$$
E_{n}=E_{n}^{-} \oplus E^{0} \oplus E_{n}^{+}, \text {where } E_{n}^{+} \subset E^{+}, E_{n}^{-} \subset E^{-} \text {for all } n
$$

$$
\left(E_{n}^{+} \text {and } E_{n}^{-} \text {are subspaces of } E\right)
$$


At least two solutions for the asymmetric beam system with critical growth 339

$$
\operatorname{dim} E_{n}<+\infty, E_{n} \subset E_{n+1}, \cup_{n \in N} E_{n} \text { is dense in } E .
$$

Let $P_{E_{n}}$ be the orthogonal projections from $E$ onto $E_{n}$.

Let us define

$$
C_{\alpha, \beta}(Q)=\inf _{(u, v) \in E \backslash(0,0)} \frac{\int_{Q} \mathcal{L} U \cdot U d x d t}{\left(\int_{Q}|u|^{\alpha}|v|^{\beta} d x d t\right)^{\frac{2}{\alpha+\beta}}} \text { for } U=(u, v)
$$

Let us prove that the functional $J$ satisfies the linking geometry.

Lemma 3.2. Assume that the conditions $(A),(B)$ and $(C)$ hold. Then

(i) there exist small numbers $\delta>0, \rho>0$ and a small ball $B_{\rho} \subset E^{+}$ with radius $\rho$ such that if $U \in \partial B_{\rho}$, then

$$
\gamma=\inf J(U) \geq \delta>0,
$$

(ii) there exist an $e \in E^{+}, R>\rho$ and a large ball $D_{R} \subset E^{0} \oplus E^{-}$with radius $R>0$ such that if

$$
W=\left(\bar{D}_{R} \cap\left(E^{0} \oplus E^{-}\right)\right) \oplus\{r e \mid 0<r<R\},
$$

then

$$
\sup _{U \in \partial W} J(U) \leq 0
$$

Proof. (i) By (C) and (3.5), for $U \in E^{+}$

$$
\begin{aligned}
& J(U) \\
& =\frac{1}{2} \int_{Q} \mathcal{L} U \cdot U-\frac{1}{2} \int_{Q}(A U, U)_{R^{2}}-\frac{2}{\alpha+\beta} \int_{Q}\left(u+u_{0}\right)_{+}^{\alpha}\left(v+v_{0}\right)_{+}^{\beta} d x d t \\
& \geq \frac{1}{2}\left(1-\frac{\sqrt{a b}}{\lambda_{00}}\right)\|U\|_{E}^{2}-\frac{2}{\alpha+\beta}\left(C_{(\alpha, \beta)}(Q)\right)^{-\frac{\alpha+\beta}{2}}\|U\|_{E}^{\alpha+\beta} .
\end{aligned}
$$

Since $\sqrt{a b}<1=\lambda_{00}$ and $\alpha+\beta>2$, there exist a number $\delta>0$, a small number $\rho>0$ and a small ball $B_{\rho} \subset E^{+}$with radius $\rho$ such that if $U \in \partial B_{\rho} \subset E^{+}$, then $\inf J(U) \geq \delta>0$.

(ii) Let us choose an element $e \in E^{+}$. Let us fix $\tilde{U}=(\tilde{u}, \tilde{v}) \neq(0,0) \in$ $E^{0} \oplus E^{-} \oplus\{r e \mid 0<r<R\}$ such that

$$
\int_{Q}(\tilde{u}-1)^{\alpha}(\tilde{v}-1)^{\beta}>0 .
$$


We note that

$$
\begin{aligned}
& \text { if } U \in E^{+} \text {, then } \int_{Q}\left(\mathcal{L} U \cdot U-(A U, U)_{R^{2}}\right) d x d t \geq \tau_{1}\|U\|_{E}^{2}, \\
& \text { if } U \in E^{-} \text {, then } \int_{Q}\left(\mathcal{L} U \cdot U-(A U, U)_{R^{2}}\right) d x d t \leq-\tau_{2}\|U\|_{E}^{2}
\end{aligned}
$$

for some $\tau_{1}>0, \tau_{2}>0$. For $s>0$ we have

$$
\begin{aligned}
& J(s \tilde{U}) \\
& \begin{array}{l}
=\frac{s^{2}}{2}\left(\int_{Q} \mathcal{L} U \cdot U d x d t-\int_{Q}(A \tilde{U}, \tilde{U}) d x d t\right) \\
\quad-\frac{2}{\alpha+\beta} s^{\alpha+\beta} \int_{Q}\left(\tilde{u}+\frac{u_{0}}{s}\right)^{\alpha}\left(\tilde{v}+\frac{v_{0}}{s}\right)^{\beta} d x d t
\end{array} \\
& \leq \frac{s^{2}}{2}\|\mathcal{L}-A\|\left\|P^{+} \tilde{U}\right\|_{E}^{2} \\
& \quad-\frac{2}{\alpha+\beta} s^{\alpha+\beta} \int_{Q}\left(\tilde{u}+\frac{u_{0}}{s}\right)^{\alpha}\left(\tilde{v}+\frac{v_{0}}{s}\right)^{\beta} d x d t-\tau_{2} \frac{s^{2}}{2}\left\|P^{-} \tilde{U}\right\|_{E}^{2} .
\end{aligned}
$$

Choosing $s_{1}>0$ such that $\frac{u_{0}(x, t)}{s_{1}}, \frac{v_{0}(x, t)}{s_{1}} \geq-1, \forall(x, t) \in Q$, we get, by (3.6) that the last integral in the equality above is positive for $s \geq s_{1}$ since $\sqrt{a b}<1=\lambda_{00}$ and $\alpha+\beta>2$. Hence $J(s \tilde{U}) \rightarrow-\infty$ as $s \rightarrow \infty$. Therefore we can choose a large number $R>0$ and a large ball $D_{R} \subset$ $E^{0} \oplus E^{-}$with radius $R>0$ such that if $W=\left(\bar{D}_{R} \cap\left(E^{0} \oplus E^{-}\right)\right) \oplus\{r e \mid 0<$ $r<R\}$, then $\sup _{U \in \partial W} J(U) \leq 0$. So the assertion (ii) hold. So the lemma is proved.

Jung and Choi [2] proved that the functional $J$ satisfies the $(P . S .)_{c}^{*}$ condition for any $c \in R$.

Lemma 3.3. Assume that the conditions $(A),(B)$ and $(C)$ hold. Then the functional $J$ satisfies the $(P . S .)_{c}^{*}$ condition with respect to $\left(E_{n}\right)_{n}$ for any real number $c$.

\section{Main result}

In this section we prove the main result of this paper. 
At least two solutions for the asymmetric beam system with critical growth 341

Theorem3.1. Assume that the conditions (A), (B) and (C) hold. Then for $(s, t)$ with $s>0$ and $t>0$ system (1.5) has at least two solutions, one of which is a positive solution $U_{0}=\left(\begin{array}{l}u_{0} \\ v_{0}\end{array}\right)$ with $u_{0}>0$ and $v_{0}>0$.

Proof. We note that $J(0,0)=0$ and $(u, v) \mapsto \operatorname{grad}\left(\frac{2}{\alpha+\beta} \int_{Q}\left(u+u_{0}\right)_{+}^{\alpha}(v+\right.$ $\left.\left.v_{0}\right)_{+}^{\beta} d x d t\right)$ is a compact mapping. By Lemma 3.2 , there exist a small number $\delta>0, \rho>0$ and a small ball $B_{\rho} \subset E^{+}$with radius $\rho$ such that if $U \in \partial B_{\rho}$, then $\gamma=\inf J(U) \geq \delta>0$, and there is an $e \in E^{+}$, $R>\rho>0$ and a large ball $D_{R}$ with radius $R>0$ such that if $W=\left(\bar{D}_{R} \cap\left(E^{0} \oplus E^{-}\right)\right) \oplus\{r e \mid 0<r<R\}$, then $\sup _{U \in \partial W} J(U) \leq 0$. Let us set $\tau=\sup _{W} J$. We note that $\tau<+\infty$. Let $\left(E_{n}\right)_{n}$ be a sequence of subspaces of $E$ satisfying (3.4). Clearly $E^{0} \subset E_{n}$ for all $n$, and $\partial B_{\rho}$ and $\partial W$ link. We have, for all $n \in N$,

$$
\gamma \leq \sup _{\partial W \cap E_{n}} J<\inf _{\partial B_{\rho} \cap E_{n}} J
$$

Moreover, by Lemma 3.3, $J_{n}=\left.J\right|_{E_{n}}$ satisfies the (P.S. $)_{c}^{*}$ condition for any $c \in R$. Thus by Lemma 3.1 (Linking Theorem), there exists a critical point $\left(u_{n}, v_{n}\right)$ for $J_{n}$ with

$$
\gamma \leq \inf _{\partial B_{\rho} \cap E_{n}} J \leq J\left(u_{n}, v_{n}\right) \leq \sup _{W \cap E_{n}} J \leq \tau
$$

Since $J_{n}$ satisfies the $(P . S .)_{c}^{*}$ condition, we obtain that, up to a subsequence, $\left(u_{n}, v_{n}\right) \rightarrow(\bar{u}, \bar{v})$, with $(\bar{u}, \bar{v})$ a critical point for $J$ such that $\gamma \leq J(\bar{u}, \bar{v}) \leq \tau$. Hence $(\bar{u}, \bar{v}) \neq(0,0)$. Thus the functional $I$ has two nontrivial solutions, one of which is a positive solution $\left(u_{0}, v_{0}\right)$ and the second solution of which is $\left(\bar{u}+u_{0}, \bar{v}+v_{0}\right)$. Thus system (1.5) has at least two solutions.

Acknowledgements The authors appreciate very much for the referee's corrections.

\section{References}

[1] Q.H. Choi, T. Jung, and P.J. McKenna, The study of a nonlinear suspension bridge equation by a variational reduction method, Appl. Anal. 50 (1993), 7190.

[2] T. Jung and Q.H. Choi, Multiple solutions for a class of the systems of the critical growth suspension bridge equations, Korean J. Math. 16 (2008), 377389. 
[3] J.Q. Liu, Free vibrations for an asymmetric beam equation, Nonlinear Anal. 51 (2002), 487-497.

[4] P.J. McKenna and W. Walter, Nonlinear oscillations in a suspension bridge, Arch. Ration. Mech. Anal. 98 (1987), no. 2, 167-177.

[5] H. Nam and Q.H. Choi, The investigation of free vibrations for an asymmetric beam equation using category theory, J. Math. Anal. Appl. 318 (2006), 552563.

Department of Mathematics

Kunsan National University

Kunsan 573-701, Korea

E-mail: tsjung@kunsan.ac.kr

Department of Mathematics Education

Inha University

Incheon 402-751, Korea

E-mail: qheung@inha.ac.kr 